\title{
EL CARIOTIPO DE CHAETANTHERA PENTACAENOIDES (PHIL.) HAUMAN (ASTERACEAE)
}

\section{THE KARYOTYPE OF CHAETANTHERA PENTACAENOIDES (PHIL.) HAUMAN (ASTERACEAE)}

\author{
Carlos M. Baeza \& Cristián Torres-Díaz \\ Departamento de Botánica, Universidad de Concepción, Concepción-Chile. cbaeza@udec.cl
}

\section{ABSTRACT}

The karyotype of Chaetanthera pentacaenoides (Phil.) Hauman from Chile was examined. This species belong to the subgenus Egania. The species has $2 \mathrm{n}=2 \mathrm{x}=20$ chromosomes, with $8 \mathrm{~m}+2 \mathrm{sm}$ chromosomes. The reported karyotype is symmetric (AsI \% $=58.73)$.

El género Chaetanthera Ruiz et Pav. es nativo de Sudamérica e incluye alrededor de 42 especies, de las cuales unas 20 ocurren en Chile (Cabrera 1937, Davies \& Facher 2001, Baeza 2005a, 2005b). El número de especies examinadas citológicamente es escaso y sólo incluye a $C$. glabrata (DC.) Meigen $(2 \mathrm{n}=28)$, C. linearis Poeppig $(2 \mathrm{n}=22), C$. linearis Poeppig var. albiflora Phil. $(2 \mathrm{n}=24), C$. tenella Less. ( $\mathrm{n}=14)$, C. microphylla (Cass.) Hook. et Arn. $(2 \mathrm{n}=24), C$. chilensis (Willd.) DC. y C. ciliata Ruiz et Pav. $(2 \mathrm{n}=22)$ (Powell et al. 1974, Grau 1987, Baeza \& Schrader 2005 a y b). Chaetanthera pentacaenoides (Phil.) Hauman es una especie perenne, alto-andina, distribuida en la Región Metropolitana de Chile y en Mendoza, Argentina.

Una de las fuentes de ayuda para resolver problemas taxonómicos es la citología y su utilidad está plenamente reconocida, sobre todo en géneros donde la morfología no es suficiente o donde la variabilidad fenotípica es muy alta (Stuessy 1990). En este trabajo se documenta el complemento cromosómico de $C$. pentacaenoides y se establece un nuevo número diploide en el género.

Se estudió una población de Chaetanthera pentacaenoides colectada en Chile, Región Metropolitana, comuna de Lo Barnechea, Valle Nevado,

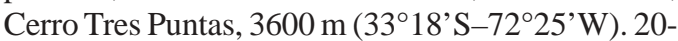

I-2003, C. Torres s.n. El material de referencia está depositado en el Herbario de la Universidad de Concepción (CONC).

El estudio de los cromosomas y mediciones se realizaron siguiendo la metodología propuesta por Baeza (1996) y Baeza et al. (2000, 2001, 2004). Para la población analizada (8 placas metafásicas) se determinó el índice de asimetría del cariotipo (AsI $\%$ ) definido por Arano \& Saito (1980). Los cromosomas se clasificaron de acuerdo a Levan et al. (1964).

Chaetanthera pentacaenoides presenta $2 \mathrm{n}=2 \mathrm{x}$ $=20$ cromosomas, con un cariotipo simétrico y una fórmula haploide $8 \mathrm{~m}+2 \mathrm{sm}$, esto es, 8 pares de cromosomas metacéntricos (pares 1, 2, 3, 5, 7, 8, 9 y 10) y dos pares de cromosomas submetacéntricos (pares 4 y 6) (Figs. 1-2). El cuociente entre el par más largo y el par más corto (R) fue 1,90. El índice de asimetría del cariotipo (AsI \%) fue de 58,73. Cabrera (1937) reconoce para Chaetanthera 7 subgéneros. C. ciliata, C. linearis, C. microphylla y C. tenella pertenecen al subgénero Chaetanthera, el cual presenta $2 \mathrm{n}=22,24,28$. $C$. chilensis está incluida en el subgénero Proselia, el cual presenta un $2 \mathrm{n}=22$. C. glabrata pertenece al subgénero Tylloma, con un $2 \mathrm{n}=28$. La especie analizada, Chaetanthera pentacaenoides, está incluida en el 
subgénero Egania, con un 2n =20, lo que representa, hasta el momento, el número diploide de cromosomas más bajo encontrado en Chaetanthera. Es probable que la disploidía haya sido uno de los mecanismos de evolución cromosómica que ocurrieron en Chaetanthera y que estarían explicando el variado número cromosómico presente en este gé- nero (Baeza \& Schrader 2005 b). La presencia de polimorfismo entre homólogos es también una situación observada en Chaetanthera, lo que ya ha sido citado para otras especies de plantas vasculares, como es el caso de Alstroemeria (Buitendjik et al. 1998), Allium (Houben et al. 2000), Placea (Baeza \& Schrader 2004), entre otras.

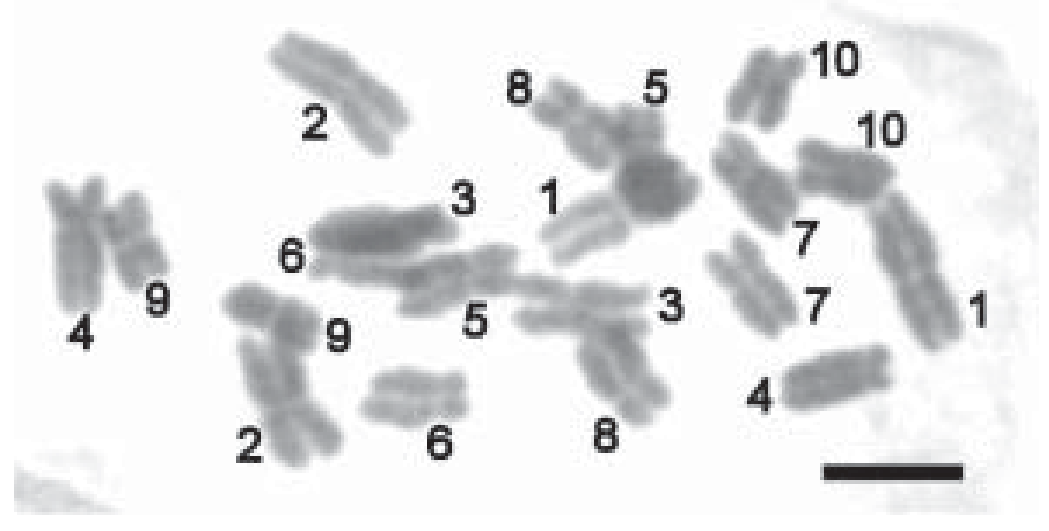

FiguRA 1. Cromosomas en metafase de Chaetanthera pentacaenoides $(2 \mathrm{n}=20)$. Escala $=5 \mu \mathrm{m}$.

FIGURE 1. Metaphasic chromosomes of Chaetanthera pentacaenoides $(2 \mathrm{n}=20)$. Scale $=5 \mu \mathrm{m}$.

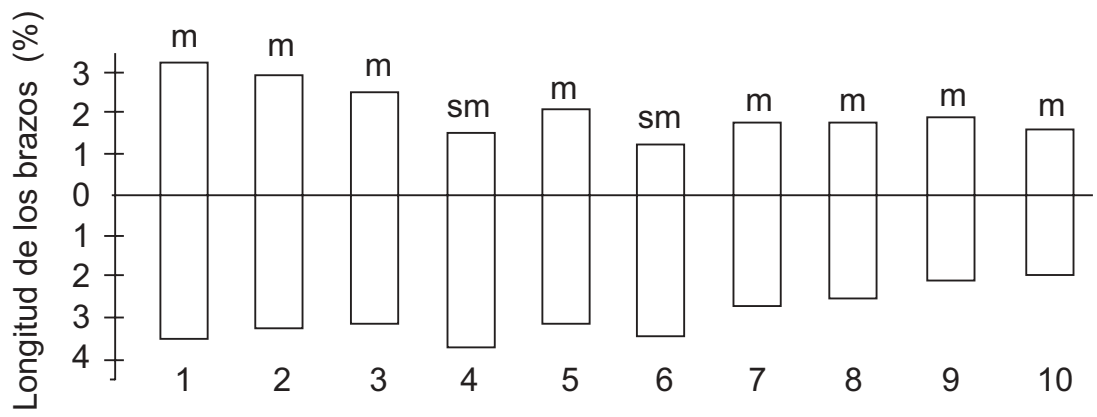

FIGURA 2. Idiograma haploide de Chaetanthera pentacaenoides $(\mathrm{m}=$ metacéntico, $\mathrm{sm}=$ submetacéntrico). Los cromosomas se han ordenado de acuerdo a su tamaño decreciente.

FIGURE 2. Ideogram haploid of Chaetanthera pentacaenoides $(\mathrm{m}=$ metacentric, $\mathrm{sm}=$ submetacentric $)$. The chromosomes have been ordered according to decreasing size. 


\section{AGRADECIMIENTOS}

Se agradece a los proyectos Fundación Andes $\mathrm{N}^{\circ} \mathrm{C}$ 14055, Fondecyt No 1020956 y 7020956 e ICM Proyecto P02-051. Cristián Torres-Díaz agradece a la Beca de Doctorado CMEB (P02-051-F).

\section{BIBLIOGRAFIA}

Arano, H. \& H. Saito. 1980. Cytological studies in family Umbelliferae 5. Karyotypes of seven species in subtribe Seselinae. La Kromosomo 2: 471-480.

BAEZA, C. 1996. Número de cromosomas en algunas especies chilenas de Danthonia DC. y Rytidosperma Steud. (Poaceae). Gayana Botánica 53(2): 329333.

Baeza, C., J. Grau, M. Vosyka, T. Stuessy \& H. Weiss. 2000. Recuentos cromosómicos en especies de Hypochaeris L. de Chile. Gayana Botánica 57(1): 105-106.

Baeza, C., G. Kottirsch, J. Espejo \& R. Reinoso. 2001. Recuentos cromosómicos en plantas chilenas. I. Gayana Botánica 58(2): 133-137.

BAEZA, C. \& O. Schrader. 2004. Karyotype analysis of Placea amoena Phil. (Amaryllidaceae) by double fluorescence in situ hybridization. Caryologia 57(2): 209-214.

BAEZA, C. \& O. Schrader. 2005 A. Análisis del cariotipo y detección de los genes $5 \mathrm{~S}$ y $18 \mathrm{~S} / 25 \mathrm{~S}$ rDNA en Chaetanthera microphylla (Cass.) H. et A. (Asteraceae). Gayana Botánica 62(1): 49-51.

BAEZA, C. \& O. SchrAdER. 2005 b. Karyotype analysis in
Chaetanthera chilensis (Willd.) DC. and Chaetanthera ciliata Ruiz et Pavón (Asteraceae) by double fluorescence in situ hybridization. Caryologia 58(4): 332-338.

Baeza, C., M. Vosyka \& T. Stuessy. 2004. Recuentos cromosómicos en plantas que crecen en Chile. II. Darwiniana 42(1-2): 25-29.

ButtendiJk, J., A. Peters, R Jan-Quené \& M. Ramanna. 1998. Genome size variation and C-band polymorphism in Alstroemeria aurea, A. ligtu and A. magnifica (Alstroemeriaceae). Plant Systematic and Evolution 212: 87-106.

CABrera, A. 1937. Revisión del género Chaetanthera (Compositae). Revista del Museo de La Plata Sección Botánica 1: 87-215.

DAvies, A. \& E. FAcher. 2001. Achene hairs and their diversity in the genus Chaetanthera Ruiz et Pav. (Mutisieae, Asteraceae). Sendtnera 7: 13-33.

Grau, J. 1987. Chromosomenzahlen chilenischer Mutisieen (Compositae). Botanische Jahrbücher 108: 229-237.

Houben, A., G. Wanner, L. Hanson, D. Verlin, C. Leach $\&$ J. Timmes. 2000. Cloning and characterization of polymorphic heterochromatic segments of Brachycome dichromosomatica. Chromosoma 109:206-213.

LeVAN, A, K. Fredga \& A. SANDBERG. 1964. Nomenclature for centromeric position on chromosomes. Hereditas 52: 201-220.

Powell, A., D. Kyhos \& P. Raven. 1974. Chromosome numbers in Compositae. X. American Journal of Botany 61: 909-913.

Stuessy, T. 1990. Plant Taxonomy. The systematics evaluation of comparative data. Columbia University Press, New York. 514 pp. 